Annuaire suisse de politique de développement

$19 \mid 2000$

Pour une politique plus cohérente envers les pays en développement

\title{
6. Politique économique extérieure
}

\section{Catherine Schümperli Younossian}

\section{(2) OpenEdition}

\section{Journals}

Édition électronique

URL : http://journals.openedition.org/aspd/853

DOI : 10.4000/aspd.853

ISSN : 1663-9669

Éditeur

Institut de hautes études internationales et du développement

\section{Édition imprimée}

Date de publication : 1 avril 2000

Pagination : 273-292

ISSN : $1660-5934$

\section{Référence électronique}

Catherine Schümperli Younossian, «6. Politique économique extérieure », Annuaire suisse de politique de développement [En ligne], 19 | 2000, mis en ligne le 19 août 2012, consulté le 07 septembre 2020 URL : http://journals.openedition.org/aspd/853 ; DOI : https://doi.org/10.4000/aspd.853 


\section{POLITIQUE ÉCONOMIQUE EXTÉRIEURE*}

D ANS CE CHAPITRE sont détaillés certains instruments autonomes de la politique économique extérieure de la Suisse (telle la GRE), ainsi que les accords économiques bilatéraux conclus entre la Suisse et certains pays en développement ou d'Europe centrale et orientale (accord de promotion et protection des investissements, de double imposition, de libre-échange, de commerce et de coopération économique). Ce chapitre établit également un inventaire des visites de chefs d'Etat en Suisse et des voyages de délégations suisses à l'étranger ayant pour objectif de tisser des relations économiques ou commerciales.

\subsection{RAPPORT SUR LA POLITIQUE ÉCONOMIQUE EXTÉRIEURE SUISSE: NOUVELLES ORIENTATIONS}

Le rapport sur la politique économique extérieure ${ }^{1}$ du 13 janvier 1999 analyse, dans son chapitre introductif, les conséquences et les enseignements à tirer de la crise asiatique du point de vue de la politique économique extérieure. Le rapport souligne que les turbulences vécues par une série de pays émergents ont montré que les efforts de la communauté internationale, comme ceux de la Suisse, ne peuvent plus avoir pour seul ou principal objectif la réalisation du «libre accès au marché». Et d'insister sur l'importance de mettre sur pied des réglementations et des institutions nationales et internationales qui devraient permettre aux marchés de fonctionner correctement.

Pour concrétiser cette proposition, le rapport dresse une liste, en six points, des directions que suivent les activités internationales afin de permettre un meilleur fonctionnement des marchés:

- La «bonne gouvernance» (good governance), notion qui relève du domaine de la politique de développement, tend à la réalisation des conditions-cadres politiques et juridiques (démocratie, Etat de droit, conduite responsable des affaires publiques, lutte contre la corruption) propres à servir de base au développement économique durable;

- l'importance d'accords de protection des investissements. Chaque Etat partie doit garantir à toute entreprise établie sur son territoire, si elle est contrôlée par un investisseur d'un autre Etat partie, l'égalité de traitement (traitement national, clause de la nation la plus favorisée), les droits rattachés à la propriété (protection contre les expropriations arbitraires) et l'accès à une juridiction internationale (arbitrage international);

- les normes fondamentales du travail inscrites dans la «Déclaration de l'OIT relative aux principes et droits fondamentaux au travail», adoptée en 1998 à Genève, sont obligatoires pour tous les Etats. Le rapport précise que si leur première raison d'être n'est pas de nature économique, elles jouent néanmoins un rôle important dans le fonctionnement durable des marchés;

* Par Catherine Schümperli Younossian, chargée de recherche à l'IUED.

1. «Rapport de la politique économique extérieure 98/1+2», Feuille fédérale, n 8, 2.3 .99 (message no 99.002). 
- la reconnaissance d'un droit efficace de la concurrence pour le bon fonctionnement des marchés, plus spécifiquement dans les économies des pays développés;

- la notion de «gouvernement d'entreprise», qui renvoie à la conduite correcte d'une entreprise, est également citée. Les propriétaires, pourvoyeurs de capitaux, employeurs, fournisseurs et clients d'une entreprise, sans oublier l'Etat, ont intérêt à ce qu'un certain nombre de règles fondamentales soient respectées dans la conduite de l'entreprise (règles de droit qui régissent les sociétés, le marché de capitaux et la comptabilité);

- le développement durable sur le plan écologique doit aujourd'hui devenir la préoccupation de tous les pays. Comme le souligne le rapport, l'un des objectifs à atteindre est également d'empêcher quiconque de s'arroger des avantages aux dépens de ses concurrents.

Après avoir établi la liste des conditions-cadres qui doivent être respectées pour que les marchés fonctionnent, le rapport souligne que la Suisse inclut les aspects sociaux et environnementaux du développement durable et qu'elle apporte son soutien aux efforts des organisations internationales économiques qui vont dans ce sens. Et de conclure en affirmant que la «politique économique extérieure» suisse est ainsi appelée à devenir de plus en plus une «politique économique mondiale» suisse.

\subsection{GARANTIE CONTRE LES RISQUES À L'EXPORTATION}

La garantie contre les risques à l'exportation (GRE) est un instrument permettant aux exportateurs suisses de conclure des contrats à l'étranger en les déchargeant de certains risques ${ }^{2}$. Fin 1998, le total des engagements s'élevait à un peu plus de 6,5 milliards de francs. Les nouvelles garanties accordées en 1998 se sont montées à 2 milliards de francs. Les ONG, suisses et internationales, ont lancé une campagne pour l'harmonisation internationale de critères sociaux et environnementaux lors de l'octroi des garanties de crédits, qui les a conduites à présenter leurs revendications à l'OCDE.

\section{$\square$ Fonctionnement général de la GRE}

Créée par la Confédération en 1934, la GRE avait pour but, à l'époque, de lutter contre le chômage. Avec les années, elle a subi des aménagements pour devenir un instrument d'encouragement des exportations suisses. Puis, sous la pression des œuvres d'entraide, en 1980, le Parlement a accepté d'introduire une disposition dans la loi sur la GRE faisant référence à la prise en compte des principes fondamentaux de la politique suisse en matière d'aide au développement lors de la décision d'octroi de garantie pour les pays les plus pauvres.

La GRE se fonde sur la Loi fédérale sur la GRE du 26 septembre 1958, sur l'ordonnance y relative du Conseil fédéral du 15 juin $1998^{3}$ et sur l'ordonnance du

2. Les risques pouvant être couverts par la GRE sont les risques politiques, de transfert, de ducroire, monétaires et liés à la livraison. On en trouve la présentation détaillée dans l'Annuaire 1999, p. 201.

3. La nouvelle ordonnance sur la GRE, avec révision des émoluments, est entrée en vigueur le $1^{\text {er }}$ juillet 1998 . Le contexte international (entrée en vigueur le $1^{\text {er }}$ avril 1999 de l'accord de l'OCDE afin d'harmoniser les primes fixées pour les assureurs étatiques) et le contenu de l'ordonnance sont détaillés dans l'Annuaire 1999 (pp. 201-202). L'harmonisation internationale permet d'éliminer des distorsions de la concurrence dont souffraient les exportateurs, en raison des barèmes de primes différents appliqués aux mêmes risques par leurs pays respectifs. 
Département fédéral de l'économie du 3 mai 1989. Comme toutes les agences de crédit à l'exportation, la GRE est soumise à des règles internationales établies dans le cadre de l'Union de Berne et de l'OCDE. L'Union de Berne, dont le siège est à Londres, rassemble les agences de crédit à l'exportation, publiques ou privées, de plus de 40 pays. Elle permet de négocier certains principes et d'assurer le respect de règles liées aux conditions de crédit, dans le cadre du commerce international. Quant à l'OCDE, son Arrangement sur les crédits à l'exportation est en vigueur depuis 1978, et a subi une modification importante en juillet 1998. Cet arrangement contient des dispositions concernant le financement et la garantie étatiques des exportations, l'harmonisation des primes minimales et le financement de l'aide liée ${ }^{4}$. Les garanties émises par les agences doivent tenir compte de ces règles.

\section{Procédure: de la demande à l'octroi de la GRE}

La garantie contre les risques à l'exportation n'est accordée qu'aux entreprises domiciliées en Suisse et inscrites au Registre du commerce. Lorsqu'un exportateur formule une demande de garantie, une procédure en plusieurs étapes est lancée, dont les principales sont présentées ci-dessous.

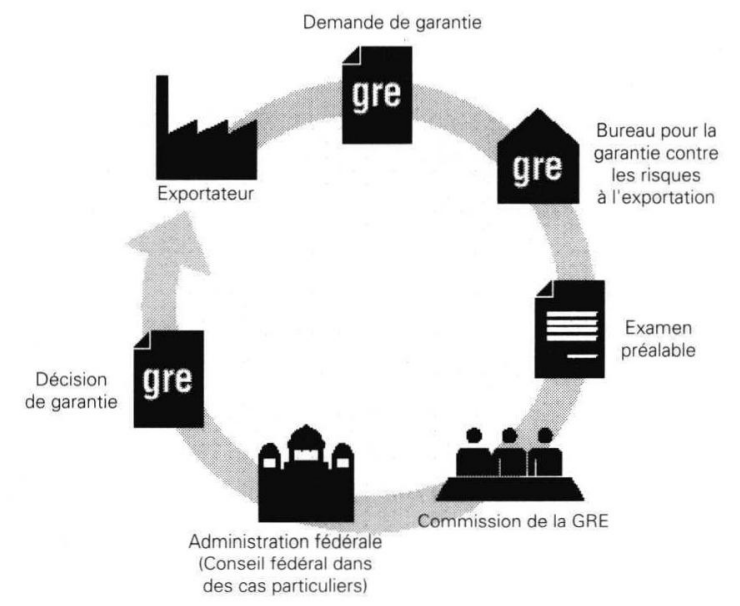

๑ Demande de principe

Préalablement à une demande formelle de garantie, l'exportateur peut se renseigner auprès du Bureau pour la GRE afin d'apprendre si, et à quelles conditions, une affaire précise peut être couverte par la GRE. Celle-ci est liée pendant six mois par son acceptation écrite pour autant que la situation demeure inchangée dans le pays acquéreur.

\section{Demande de garantie}

Indépendamment d'une éventuelle demande de principe et une fois le contrat conclu, l'exportateur requiert l'octroi d'une garantie.

\section{- Indications complémentaires}

En ce qui concerne les livraisons dans les pays en voie de développement les plus pauvres, un questionnaire supplémentaire doit être rempli, lequel porte sur l'utilisation, la viabilité économique, les aspects écologiques, la formation, etc. 


\section{口 Date de la demande}

Les demandes de garantie peuvent être déposées au plus tôt trois mois avant l'entrée en risque, mais jamais après que le risque a commencé

\section{- A qui adresser la demande?}

Les questions et les demandes de garantie doivent être adressées au Bureau pour la GRE (Zurich). Pour simplifier la procédure administrative, certaines associations accordent des garanties dites globales pour le secteur de l'industrie qu'elles représentent (telles la Société suisse de l'industrie chimique SSIC, la Industrie- und Handelskammer pour les textiles, et la Fédération de l'industrie horlogère suisse FH).

- Obligations du bénéficiaire de la garantie

L'exportateur ou le cessionnaire doit informer le Bureau pour la GRE des paiements contractuels effectués de l'étranger. II doit également immédiatement informer la GRE lorsqu'une échéance contractuelle n'a pas été payée dans les délais ou qu'une perte paraît imminente. En principe, le bénéficiaire de la garantie doit, en accord avec la GRE, prendre toutes les mesures propres à limiter les dommages.

\section{口 Procédure}

Après avoir procédé à un examen préalable, le Bureau pour la GRE soumet les demandes à la Commission de la GRE, qui siège tous les quatorze jours. Cette commission se compose de huit membres, dont quatre font partie de l'administration fédérale et quatre représentent les milieux économiques; ils sont nommés par le Conseil fédéral. Sur la base des propositions de la commission, c'est le Secrétariat d'Etat à l'économie (Seco) qui décide de l'octroi de la GRE lorsque le montant n'excède pas 5 millions de francs. Les garanties de plus de 10 millions de francs doivent en outre recevoir le consentement du Département fédéral des finances. Les garanties d'une portée particulière ou politiquement délicates doivent avoir l'aval du Conseil fédéral. Lorsqu'une garantie est accordée, le Seco notifie la décision à l'exportateur. En cas de rejet de la demande de garantie par la commission, ou lorsque le requérant n'est pas d'accord avec le contenu de la décision de garantie, il peut déposer un recours administratif.

Source: site Internet du Bureau pour la GRE (www.swiss-erg.com).

\section{$\square$ Fonds indépendant de la Confédération}

La GRE n'est pas un instrument de coopération au développement, mais un fonds institué par la Confédération, financièrement indépendant. Les dépenses et les recettes de la GRE ne figurent donc pas dans le compte financier de la Confédération. En vue d'aboutir, à long terme, à son autonomie financière, la GRE facture aux exportateurs demandant une garantie un émolument lié au risque. L'émolument est fonction du risque du pays ${ }^{5}$, du montant, de la durée et des autres risques couverts.

Tableau 18: Aperçu de l'évolution des activités de la GRE (1996-1998)

\begin{tabular}{lrrr}
\hline Année & $\mathbf{1 9 9 6}$ & $\mathbf{1 9 9 7}$ & $\mathbf{1 9 9 8}$ \\
\hline Garanties en vigueur (nombre) & 1521 & 1471 & $\mathbf{1 2 6 3}$ \\
\hline Nouvelles garanties (mio. fr.) & 1424 & 2480 & $\mathbf{2 0 1 1}$ \\
\hline Engagements (mio. fr.) & 5941 & 6421 & $\mathbf{6 5 3 7}$ \\
\hline Emoluments nets (mio. fr.) & 43 & 94 & $\mathbf{3 7}$ \\
\hline Indemnités versées (mio. fr.) & 108 & 53 & $\mathbf{4}$ \\
\hline Résultats annuels (mio. fr.) & 23 & 213 & $\mathbf{1 9}$ \\
\hline Excédents/pertes à reporter (mio. fr.) & -704 & -491 & $\mathbf{4 7 2}$ \\
\hline Provisions (mio. fr.) & 700 & 999 & $\mathbf{9 7 9}$ \\
\hline
\end{tabular}

Source: Bureau pour la GRE, OFAEE, Rapport annuel 1998 de la GRE، Zurich/Berne, juin 1999, annexe A, p. 22.

5. La GRE a établi une classification sur une échelle de 1 (risques minimes) à 7 (risques importants) des pays pour lesquels une garantie peut être demandée. Cette liste est consultable sur le site Internet de la GRE. 
Il se dégage du compte du résultat que cette année encore, la GRE peut subvenir à ses besoins (excédent de 19 millions). Pour la quatrième année consécutive, elle a affiché un excédent de recettes. En 1998, elle a remboursé 251 millions de francs, ce qui porte, depuis 1995, à 688 millions de francs le total de l'amortissement des avances de la Confédération, de sorte que celles-ci sont maintenant réduites à 897 millions de francs, sans intérêts jusqu'à la fin $1999^{6}$. Comme les années précédentes, l'évolution du nombre de sinistres a été favorable. Les crises financières qui ont secoué l'Asie et la Russie de même que les signes de faiblesse donnés par l'Amérique latine n'ont pas entraîné, dans le courant de l'année 1998, des suppressions de paiement.

\section{$\square$ Réassurance de la Confédération}

En raison du principe d'autonomie qui régit la GRE et selon les circonstances, il n'est pas possible d'accorder de garantie pour certains pays. Néanmoins, afin de permettre à l'exportateur d'obtenir une garantie malgré cela, le Parlement a accepté des programmes spéciaux dans lesquels la Confédération supporte directement le risque; cela s'applique notamment pour les pays de l'Est ${ }^{7}$. Le Bureau pour la GRE se charge de la gestion administrative des dossiers.

En 1998, la Commission de la GRE a décidé qu'elle se prête à nouveau à la garantie de crédits commerciaux afférents à des projets d'investissements réalisés dans la plupart des pays d'Europe centrale et du Sud-Est. Il n'est donc plus nécessaire de couvrir ces risques par le biais de l'aide à l'Europe de l'Est. Quelques exceptions doivent cependant être signalées: la Bulgarie (pour les crédits à long terme), la Macédoine, la Russie, l'Ukraine et, en Asie centrale, le Kazakhstan et l'Ouzbékistan bénéficient encore des garanties de crédits de la Confédération. Chaque année, une évaluation est faite de la situation économique et politique des pays concernés de sorte que la liste des pays bénéficiant de la réassurance de la Confédération est revue périodiquement. A noter également que la plupart des pays africains sont exclus de la GRE pour l'octroi de crédits à moyen et à long terme. En cas de demande de couverture d'exportateurs suisses pour l'Afrique, une réassurance de la Confédération peut être attribuée par le service de développement du Seco. Dans un tel cas, le Seco n'attribuera une réassurance que si la dimension «développement» du projet pour lequel la réassurance est demandée est explicite.

\section{Nouvelles garanties accordées en 1998}

Les nouvelles garanties accordées pour un montant total d'environ 2 milliards de francs ont baissé de près de $20 \%$ par rapport à l'année précédente (environ 2,5 milliards). Cela s'explique notamment par le fait que seuls deux grands projets, d'une valeur supérieure à 50 millions de francs, ont été couverts au cours de l'exercice considéré. Comme en 1997, le plus grand nombre de garanties a été octroyé pour la Turquie (couvrant environ 450 millions de francs). La Chine, l'Iran ainsi que la Grèce (avec un grand projet) font partie des pays pour lesquels un volume important de garanties a été accordé.

6. «Rapport de la politique économique extérieure 98/1+2», Feuille fédérale, n 8, 2.3.99 (message n 99.002).

7. Dans le cadre du programme de coopération renforcée avec les pays de l'Europe de l'Est, le Conseil fédéral a alloué un cadre de garantie, c'est-à-dire une réassurance de la Confédération, en faveur des livraisons de marchandises et de la prestation de services suisses. 
Tableau 19: Répartition géographique des nouvelles garanties et du total des engagements de la GRE en 1998

\begin{tabular}{lrrrr}
\hline & $\begin{array}{c}\text { Nouvelles garanties } \\
\text { en 1998 }\end{array}$ & \multicolumn{2}{c}{$\begin{array}{c}\text { Total des engagements } \\
\text { au 31.12.1998 }\end{array}$} \\
\hline Groupes de pays & $\begin{array}{c}\text { Millions } \\
\text { de fr. }\end{array}$ & $\begin{array}{c}\text { part } \\
\text { en \% }\end{array}$ & $\begin{array}{r}\text { Millions } \\
\text { de fr. }\end{array}$ & $\begin{array}{r}\text { part } \\
\text { en \% }\end{array}$ \\
\hline Europe (sans Europe cent. et orient.) & 641.7 & 31.9 & 1467.2 & 22.4 \\
\hline Pays d'Europe centrale et orientale & 114.7 & 5.7 & 251.5 & 3.8 \\
\hline Amérique centrale et du Sud & 198.7 & 9.9 & 591.9 & 9.1 \\
\hline Afrique & 109.7 & 5.5 & 384.0 & 5.9 \\
\hline Moyen-Orient & 382.7 & 19.0 & 1391.3 & 21.3 \\
\hline Autres pays d'Asie & 563.2 & 28.0 & 2451.6 & 37.5 \\
\hline Total & $\mathbf{2 0 1 0 . 7}$ & $\mathbf{1 0 0 . 0}$ & $\mathbf{6 5 3 7 . 5}$ & $\mathbf{1 0 0 . 0}$ \\
\hline dont: 72 pays les plus défavorisés & 382.8 & 19.0 & 1262.6 & 19.3 \\
\hline \multicolumn{1}{c}{ dont: Amérique } & 0.0 & 0.0 & 0.8 & 0.0 \\
\hline \multicolumn{1}{c}{ Afrique } & 40.4 & 2.0 & 114.0 & 1.7 \\
\hline \multicolumn{1}{c}{ Asie } & 342.4 & 17.0 & 1147.8 & 17.6 \\
\hline
\end{tabular}

Source: Bureau pour la GRE, OFAEE, Rapport annuel 1998 de la GRE, Zurich/Berne, juin 1999, tableau de l'annexe C/1, p. 24.

Les garanties accordées en 1998 se concentrent principalement sur deux branches économiques, la chimie et les machines. Une nouvelle méthode de comptabilisation des garanties globales a entraîné une hausse de la part de la chimie de $14 \%$ à $54 \%$ et celle des machines a chuté de $83 \%$ à $45 \%$. Les autres secteurs professionnels (biens de consommation, ingénierie, bâtiment) ne sollicitent pratiquement pas la GRE.

$\square$ Total des engagements de la GRE

Graphique 2: Les 15 principaux pays couverts par la GRE Montant total des engagements au 31.12.1998, en millions de francs

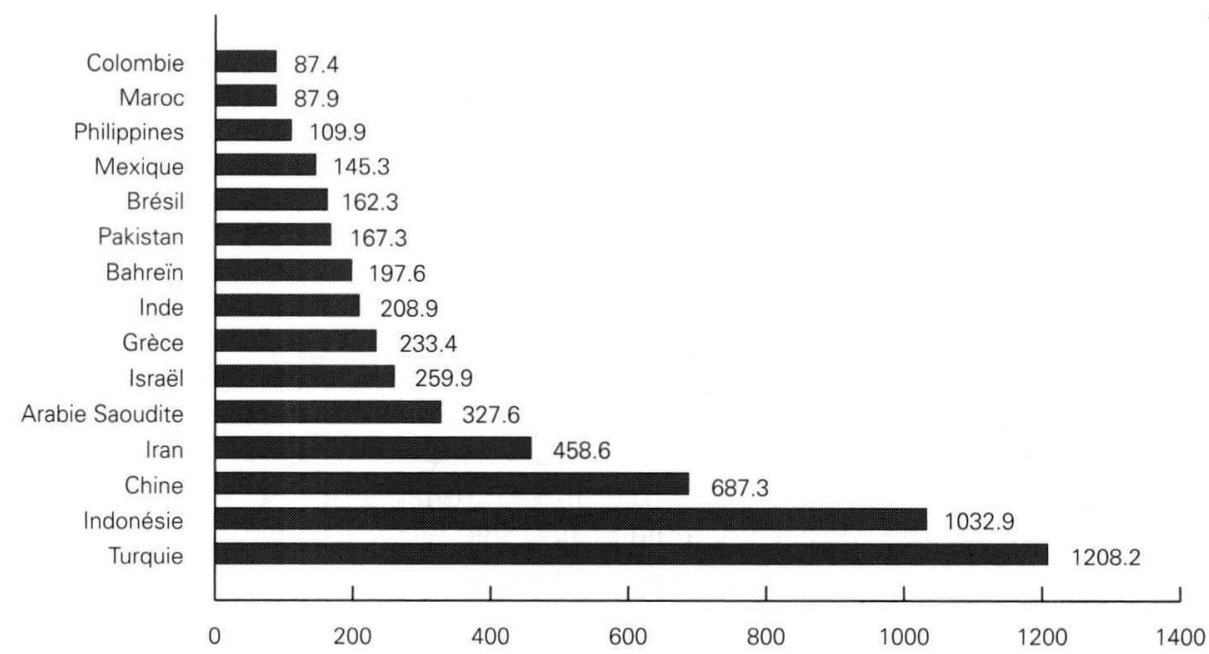

Source: Bureau pour la GRE, OFAEE, Rapport annuel 1998 de la GRE, Zurich/Berne, juin 1999, tableau de l'annexe F/1-2-3, pp. 30-32. 
Le total des engagements s'élevait à un peu plus de 6,5 milliards fin 1998. Le graphique 2 relève les 15 principaux pays bénéficiaires, qui correspondent à $84,5 \%$ du total des engagements de la GRE. La Turquie, l'Indonésie, la Chine et l'Iran représentent ensemble 53,3\% de tous les engagements. Au cours de l'exercice 1998, la Colombie, le Maroc et la Grèce ont remplacé l'Egypte, l'Argentine et l'Algérie.

En principe, les garanties peuvent être accordées pour les pays qui bénéficient déjà d'engagements importants, tant que ces pays sont solvables et qu'ils n'engendrent pas d'indemnités à payer. Si des difficultés de paiement apparaissent, qui nécessiteraient des indemnisations, des mesures seront prises par la Commission de la GRE pour empêcher un accroissement des engagements. Cette situation s'est produite pour l'Indonésie, qui a dû solliciter de l'aide auprès du FMI et des banques de développement, et demander un rééchelonnement au Club de Paris en raison des difficultés économiques auxquelles elle est confrontée.

\section{$\square$ Pays en développement les plus défavorisés}

Comme l'indique le tableau 19, le groupe des pays les plus défavorisés s'est vu octroyer des nouvelles garanties pour 382,8 millions de francs, correspondant aux 19\% des nouvelles garanties accordées en 1998. Cette répartition est toutefois très inégale entre les trois continents. L'Asie représente la part la plus importante de ce groupe avec 342 millions de nouvelles garanties et un engagement total de 1,1 milliard de francs; elle est suivie par l'Afrique, qui a bénéficié de 40 millions de francs de garanties (pour un engagement total de 114 millions).

\section{$\square$ Position des ONG suisses sur la GRE en 1999}

Durant l'année 1999, les organisations non gouvernementales ont suivi de manière très attentive le dossier de la GRE. Leurs prises de position ont porté principalement sur l'harmonisation internationale des critères environnementaux pour l'attribution de crédits à l'exportation, ainsi que sur les nouvelles garanties accordées aux entreprises suisses.

\section{Harmonisation internationale \\ des critères environnementaux pour l'attribution de crédits à l'exportation}

En 1998, une déclaration d'intention a été conclue à l'OCDE, reconnaissant la nécessité de prendre en compte les facteurs environnementaux lors de l'octroi des garanties de crédits à l'exportation. Dans ce contexte, un accord a été signé sur l'échange d'informations environnementales concernant les grands projets (tels le barrage des Trois-Gorges, en Chine, ou celui d'Ilisu, en Turquie). Selon l'accord, une étude d'impact sur l'environnement doit être fournie par l'acheteur et transmise à tous les organismes de crédit à l'exportation, qui sont tenus de faire connaître leurs vues et préoccupations suscitées par le projet dans l'optique de l'environnement ${ }^{8}$.

Selon le Rapport annuel 1998 de la GRE, la Suisse a été la première à faire appel à la reconnaissance contenue dans cette déclaration, c'est-à-dire à une coopération renforcée autour du projet, dans le cadre de la construction d'une usine hydraulique en Turquie. Les GRE de huit pays se coordonnent pour trouver un

8. Site de l'OCDE consacré aux crédits à l'exportation: www.oecd.org./ech/act/xcred-fr.htm. 
dénominateur commun lors de plusieurs rencontres en vue de déterminer leur position face à ce projet ${ }^{9}$.

Les ONG de développement et d'environnement, tant dans les pays industrialisés que dans les pays du Sud, revendiquent depuis des années l'intégration de standards sociaux et environnementaux dans l'attribution des garanties de crédits. Afin de coordonner leurs efforts, elles se sont regroupées au sein d'une coalition internationale et ont lancé une campagne au niveau mondial pour dénoncer les garanties étatiques qui soutiennent des projets destructeurs dans les pays en développement: mégabarrages, centrales thermiques, prospection pétrolière en forêt vierge. La Déclaration de Berne et l'organisation non gouvernementale américaine Environmental Defense Fund animent et coordonnent la coalition. Selon celle-ci, depuis 1996, les agences gouvernementales de crédit et de garantie à l'exportation ont appuyé leurs entreprises nationales pour plus de 100 milliards de dollars par an. Ainsi, en Europe occidentale, en Amérique du Nord, au Japon et en Australie, de telles agences (export credit agencies - ECA) soutiennent plus de $10 \%$ du commerce mondial, sous forme de prêts, de garanties et d'assurances. Et le représentant du Fonds de défense environnementale de souligner que les ECA financent souvent des projets que la Banque mondiale ou d'autres agences nationales et internationales ont rejetés pour des raisons non seulement socio-écologiques, mais aussi économiques ${ }^{10}$.

Les ONG dénoncent la concurrence malsaine entre les Etats qui respectent certains critères et d'autres qui en profitent. Elles prennent l'exemple des ECA américaines qui, sous leur pression, ont récemment introduit des normes sur les droits de l'homme et l'environnement et de ce fait ont perdu des marchés (Trois-Gorges et Ilisu). Les ECA allemande, suisse, canadienne, suédoise, française, espagnole et brésilienne, notamment, n'ont pas ce type de critères, ce qui a permis à certaines d'entre elles de remporter les marchés. Cette situation a poussé des députés républicains au Congrès américain à réclamer la suppression des normes qui affaiblissent la compétitivité de leur pays.

Dans ces conditions, une harmonisation internationale des critères sociaux et environnementaux s'impose. La Déclaration d'intention et l'accord signé en 1998 à l'OCDE (voir ci-dessus) ont ouvert une première voie dans ce sens. Le Groupe de l'OCDE sur les garanties de crédits poursuit la réflexion et, dans cette optique, a auditionné les deux organisations leaders de la coalition en octobre 1999. Les délégués des ONG ont déploré que de trop nombreuses agences de crédit à l'exportation n'aient pas de lignes directrices en matière d'impact sur l'environnement et les conséquences sociales des projets soutenus et aient une politique d'information très peu transparente. Les ONG relèvent également que les effets sociaux sont peu pris en compte et que les populations concernées par ce type de projet devraient être consultées lors du lancement du projet ${ }^{11}$.

Nouvelle demande de GRE destinée au barrage des Trois-Gorges

La filiale suisse du groupe ABB a déposé, en septembre 1999, une nouvelle demande de garantie dans le cadre du projet de barrage des Trois-Gorges, en

9. Bureau pour la GRE, OFAEE, Rapport annuel 1998 de la GRE, Zurich/Berne, juin 1999, p. 1.

10. «Cessons de polluer au Sud aux frais de nos contribuables», Le Courrier, pages Liberté, 5.4.99.

11. Erklärung von Bern, «Internationale Reformen für Exportrisikogarantien gefordet» et «NGO Recommendations regarding the Environmental Mandate of the OECD Working Party on Export Credits and Credit Guarantees», Pressemitteilung, 26.10.99. 
Chine. Il s'agit, dans ce cas, de la construction d'un relais électrique de haute tension. La multinationale helvético-suédoise, qui a déjà bénéficié en octobre 1997 d'une GRE de 390 millions de francs, a présenté une demande de garantie complémentaire pour un montant de 170 millions de francs. Se basant sur la décision antérieure du Conseil fédéral et considérant qu'aucun paramètre qui entrait en ligne de compte lors de l'attribution de la GRE en 1996 n'avait fondamentalement changé, la commission a donné son accord de principe ${ }^{12}$. La Déclaration de Berne a vivement réagi à cette décision, estimant qu'elle ne tient pas compte de l'impact négatif du projet.

\author{
SOURCES \\ Bureau pour la GRE, OFAEE, Rapport annuel 1998 de la GRE, Zurich/Berne, juin 1999. \\ «Rapport de la politique économique extérieure 98/1+2», Feuille fédérale, n 8, 2.3 .99 (message n० 99.002). \\ G. Perroulaz, «GRE, à la recherche de la cohérence», Vers un développement solidaire, n 148, décembre 1998, Décla- \\ ration de Berne. \\ SITES INTERNET \\ Bureau pour la GRE: www.swiss-erg.com. \\ Déclaration de Berne (important dossier sur la GRE, en allemand uniquement): www.evb.ch/erg.htm. \\ OCDE (page principale des crédits à l'exportation): www.oecd.org./ech/act/xcred-fr.htm. \\ ADRESSE \\ Bureau pour la garantie contre les risques à l'exportation, Kirchenweg 8, 8032 Zurich. Tél. 01/384.47.77, fax 01/384.47.87, \\ e-mail office@erg.admin.ch.
}

\title{
6.3. ACCORDS ÉCONOMIQUES BILATÉRAUX
}

\section{$\square$ Accords de promotion et de protection des investissements}

En l'absence d'un cadre juridique international de protection des investissements dans un pays étranger, la Suisse a tissé un réseau très dense d'accords bilatéraux relatifs à la protection et à l'encouragement des investissements de capitaux. Par ces accords, la Confédération souhaite assurer aux entreprises suisses une sécurité juridique quand elles investissent à l'étranger. Les accords doivent garantir aux investisseurs suisses un traitement juste et équitable, conforme au droit international, sur le territoire de l'autre partie contractante. Ce traitement doit être le même que celui qui est réservé par cette partie contractante à ses propres ressortissants ou, s'il est plus favorable, aux investissements de la nation la plus favorisée. Il faut que soit garanti le transfert des revenus des investissements, tels que gains et dividendes, ou des autres paiements afférents aux investissements de capitaux. Une éventuelle dépossession doit donner lieu à une complète indemnisation et les normes de droit international doivent être respectées ${ }^{13}$. La protection des investissements englobe toutes les catégories d'avoirs dans l'autre pays: propriété de biens et d'immeubles, actions, parts sociales et autres formes de participation dans des sociétés, concessions, droits d'auteurs, droits de propriété industrielle (brevets, dessins ou modèles industriels, marques, noms commerciaux). Enfin, en cas de différend entre les parties contractantes, il faut pouvoir recourir à un tribunal arbitral.

12. Seco, «GRE destinée à la centrale des Trois-Gorges en Chine», Communiqué de presse, 20.9.99.

13. Le contenu général des accords de protection et de promotion des investissements est détaillé dans le «Message sur la prorogation de l'arrêté fédéral concernant la conclusion de traités relatifs à la protection et à l'encouragement des investissements de capitaux» du 20 octobre 1993 (message $\mathbf{n}^{\circ}$ 93.086). 
En novembre 1999, la Suisse avait signé 90 accords de promotion et de protection des 1 et investissements, dont 82 sont en vigueur avec 29 pays africains, 20 pays asiatiques, 17 pays d'Europe centrale et orientale, 15 pays d'Amérique latine et 1 pays européen (Malte) ${ }^{14}$.

En 1998, des accords de protection des investissements ont été conclus avec l'Arménie, 1'Ethiopie, le Botswana, la République populaire démocratique de Corée, les Emirats arabes unis, l'Iran, le Koweït, Maurice et le Nicaragua. En 1999, la Suisse a signé un accord avec le Kirghizistan.

\section{$\square$ Accords de double imposition}

Les pays signataires d'une convention de double imposition abandonnent une partie de leur souveraineté en matière fiscale afin d'éviter que des revenus ne soient imposés doublement, dans les deux pays concernés. Ces conventions bilatérales règlent l'imposition des personnes physiques et des entreprises: revenus, revenus immobiliers, dividendes, intérêts de créances, redevances, gains en capital, revenus d'artistes et de sportifs, pensions et impôts sur la fortune (lorsque ces derniers existent dans les deux pays). L'élimination de la double imposition est dans l'intérêt des contribuables et assure aussi une protection fiscale aux entreprises suisses actives à l'étranger. Une fois signée par les deux Etats, la convention doit être approuvée par le Parlement ${ }^{15}$. La décision n'est pas soumise au référendum facultatif.

En 1999, sept nouveaux accords de double imposition ont été signés avec l'Arabie Saoudite, le Belarus, la Croatie, le Kazakhstan, le Koweït, la République de Moldova et la Mongolie) ${ }^{16}$. Selon l'état au $1^{\text {er }}$ novembre 1999, la Suisse a signé des accords de double imposition avec 23 pays industrialisés et 25 pays en développement (dont 21 sont déjà en vigueur), et 12 avec les pays d'Europe centrale et orientale (dont 8 sont en vigueur). De plus, des négociations sont en cours avec les pays suivants: Albanie (projet paraphé), Allemagne (imposition des dividendes), Australie (révision de la convention de 1980), Géorgie, Inde (protocole paraphé), Israël, Kirghizistan, Macédoine (projet paraphé), Ouzbékistan, Pakistan (révision de la convention 1959/62, projet paraphé), Turquie, Ukraine ${ }^{17}$.

Lors de la session d'été 1999, le Parlement a discuté de la convention de double imposition avec les Philippines et a autorisé le Conseil fédéral à échanger les instruments de ratification. La Suisse figure depuis longtemps parmi les investisseurs étrangers les plus importants aux Philippines, qui représentent ainsi pour elle un partenaire économique relativement important. La convention offre une certaine protection sur le plan fiscal aux entreprises suisses qui y sont actives. Elle favorise de nouveaux investissements et contribue à ce que les entreprises suisses présentes sur le marché philippin ne subissent pas de désavantages fiscaux par rapport à leurs concurrentes d'autres pays industrialisés ${ }^{18}$.

14. La liste complète des pays dont les accords de promotion des investissements sont en vigueur est disponible sur le site Internet du Seco www.seco.adminch/entwicklung/f-inves/isa.htm

15. Les messages du Conseil fédéral demandant aux Chambres fédérales d'approuver la ratification de conventions de double imposition sont publiés dans la Feuille fédérale.

16. Trois des sept conventions de double imposition ont déjà fait l'objet d'un débat parlementaire (République de Moldova: voir Feuille fédérale, nº 33, 24.8.99; Koweït et Croatie: voir Feuille fédérale, n 37, 21.9.99).

17. Administration fédérale des contributions, liste des pays avec lesquels la Suisse a signé une convention de double imposition (état au $\mathrm{I}^{\mathrm{er}}$ novembre 1999).

18. Conseil national, Bulletin officiel de l'Assemblée fédérale, session été 1999. 


\section{Accords de libre-échange (AELE)}

Depuis 1990, les pays de l'AELE'19 (Association européenne de libre-échange) ont conclu des accords de libre-échange avec 14 Etats de l'Europe centrale et orientale ainsi que du bassin méditerranéen. Ces dernières années, plusieurs de ces accords ont été adaptés aux nouvelles règles de l'OMC, au développement des relations extérieures de l'Union européenne et aux changements intervenus au sein de l'AELE ${ }^{20}$. Les accords de libre-échange conclus avec des pays tiers ont pour but essentiel d'offrir aux entreprises exportatrices vers ces pays les mêmes conditions que celles dont bénéficient les entreprises de l'Union européenne.

Les Etats de l'AELE ont intensifié leurs efforts pour mettre en place des relations de libre-échange avec leur partenaires commerciaux du bassin méditerranéen (voir encadré ci-dessous). Le 30 novembre 1998, les représentants de l'AELE ont signé un accord de libre-échange avec l'OLP agissant pour le compte de l'Autorité palestinienne ${ }^{21}$.

Des déclarations de coopération ont été signées avec l'Albanie, l'Egypte, la Tunisie la Macédoine, la Jordanie et le Liban. Ces déclarations ont, à terme, pour objectif la conclusion d'accords de libre-échange. Les négociations sont bien avancées avec l'Egypte, la Jordanie et la Tunisie; celles avec Chypre se sont poursuivies. A noter, que pour la première fois, les Etats de l'AELE se sont lancés dans des négociations avec un partenaire d'outre-Atlantique, le Canada, en vue de mettre sur pied un régime de libre-échange.

\section{Accords de libre-échange entre l'AELE et les pays tiers}

$\begin{array}{ll}\text { Pays } & \begin{array}{l}\text { Date d'entrée } \\ \text { en vigueur de l'accord }\end{array} \\ \text { Turquie } & 1^{\text {er }} \text { octobre } 1992 \\ \text { Israël } & 1^{\text {er janvier } 1993} \\ \text { Rép. tchèque } & 23 \text { avril } 1993 \\ \text { Slovaquie } & 23 \text { avril } 1993 \\ \text { Hongrie } & 20 \text { décembre } 1993 \\ \text { Roumanie } & 30 \text { mai } 1994 \\ \text { Bulgarie } & 1^{\text {er juin } 1994}\end{array}$

$\begin{array}{ll}\text { Pays } & \begin{array}{l}\text { Date d'entrée } \\ \text { en vigueur de l'accord }\end{array} \\ \text { Lettonie } & 4 \text { juin } 1997 \\ \text { Lituanie } & 6 \text { juin } 1997 \\ \text { Pologne } & 27 \text { octobre } 1997 \\ \text { Slovénie } & \text { 1 }^{\text {er }} \text { septembre } 1998 \\ \text { Estonie } & \text { 1 }^{\text {er }} \text { octobre } 1998 \\ \text { Maroc } & \text { 1er }^{\text {er }} \text { décembre } 1999\end{array}$

Date de signature de l'accord (pas encore entré en vigueur)

Palestine (OLP) 30 novembre 1998

Source: site Internet de I'AELE www.efta.int/structure/main/index.html (EFTA's third-country relations).

19. Depuis 1995, l'AELE ne regroupe plus que 4 pays: la Suisse, la Norvège, l'Islande et le Liechtenstein.

20. Les modifications concernent les accords conclus avec la Bulgarie, Israël, la Roumanie, la Pologne, la Slovaquie, la République tchèque, la Turquie et la Hongrie ( «Message concernant la modification de divers accords de libreéchange conclus entre les Etats membres de l'AELE et des Etats tiers» du 13 janvier 1999, Feuille fédérale, n 8, 2.3.99 (message $\mathrm{n}^{\circ}$ 99.002).

21. «Arrêté fédéral portant sur l'approbation de l'accord intérimaire entre les pays de l'AELE et l'OLP agissant pour le compte de l'Autorité palestinienne. Annexe au Rapport sur la politique économique extérieure $98 / 1+2 》$ et «Message concernant les accords économiques internationaux», Feuille fédérale, no 8, 2.3.99 (message no 99.002). 


\section{$\square$ Accords de commerce et de coopération économique}

République d'Arménie

Un accord de commerce et de coopération économique entre la Suisse et la République d'Arménie a été signé le 19 novembre 1998 à Berne et adopté par les Chambres fédérales en $1999^{22}$. Il a pour but de promouvoir et de renforcer les relations économiques bilatérales et d'appuyer le processus de réforme engagé en Arménie en vue d'instaurer une économie de marché. L'accord se fonde sur les principes de l'OMC, il contient des dispositions détaillées sur la protection de la propriété intellectuelle et réglemente le domaine de la coopération économique. Valable pour une première période de cinq ans, il peut être prorogé de cinq ans en cinq ans à moins d'être dénoncé.

Les échanges commerciaux entre la Suisse et l'Arménie sont encore peu développés. En 1997, les exportations atteignaient 1,3 million de francs (essentiellement des produits agricoles, chimiques et pharmaceutiques) et les importations 4,2 millions de francs. L'Arménie bénéficie de l'aide suisse par le biais de la Banque mondiale. L'accord bilatéral de promotion et de protection des investissements signé le 19 novembre 1998 devrait faciliter les investissements directs de la Suisse en Arménie. Il s'agit d'un accord-cadre susceptible d'être développé ${ }^{23}$.

\section{Géorgie}

Le conseiller fédéral Pascal Couchepin et la ministre du Commerce et des Relations économiques extérieures de Géorgie ont signé, le 11 mars 1999 à Berne, un accord de commerce et de coopération économique. Ce premier accord économique entre la Suisse et la Géorgie place les échanges de marchandises entre les deux pays sous le signe des principes fondamentaux du GATT (clause de la nation la plus favorisée, non-discrimination, traitement national). Il contient en outre des dispositions visant à améliorer la protection de la propriété intellectuelle et précise les domaines où la coopération économique s'exercera en priorité. En 1998, la Suisse a exporté vers ce pays des biens pour une valeur d'environ 4,3 millions de francs.

\section{Corée du Sud}

La Suisse et la Corée du Sud ont institutionnalisé leur coopération économique et commerciale par la signature d'un arrangement correspondant le 29 juin 1999. Cet instrument avait été paraphé lors de la visite de Pascal Couchepin en Corée du Sud en février de la même année. L'accord couvre un vaste programme de travail conjoint ayant notamment pour objectifs la libéralisation des échanges commerciaux ainsi que la promotion du commerce et des investissements. A ce titre, il instituera un groupe consultatif afin d'explorer les possibilités de négocier des accords bilatéraux, notamment un accord de libre-échange ainsi qu'un accord de reconnaissance mutuelle en matière d'évaluation de la conformité des prescriptions. La question d'une coopération accrue en matière de procédures douanières sera également examinée. Une collaboration plus étroite entre les deux pays est aussi prévue sur le plan multilatéral, en particulier au niveau de l'Organisation mondiale du commerce.

22. «Message concernant l'accord de commerce et de coopération économique conclu entre le Conseil fédéral et le gouvernement de la République d'Arménie» du 13 janvier 1999, Feuille fédérale, n 8, 2.3.99 (message n 99.002).

23. La Suisse a déjà signé des accords similaires avec la Russie, l'Ukraine, l'Ouzbékistan, le Kazakhstan, le Belarus, la Moldavie et le Kirghizistan, qui sont tous en vigueur. 
Pour la Suisse, cet arrangement avec la Corée du Sud est le premier du genre conclu avec un pays asiatique. Il concrétise la politique du Conseil fédéral visant un resserrement des relations économiques entre la Suisse et ses partenaires extra-européens.

\section{Tadjikistan}

Le 19 octobre 1999, Remo Gautschi, vice-directeur de la DDC, et Davlat Usmon, ministre de l'Economie du Tadjikistan, ont signé à Douchanbe un accord de coopération technique et financière ainsi que d'aide humanitaire. Cet accord, qui règle les modalités de l'aide suisse pour le processus de réforme au Tadjikistan, entre en vigueur pour une première période de cinq ans. Il s'agit du tout premier accord de coopération signé par le Tadjikistan. Outre la Pologne, l'Azerbaïdjan, le Kirghizistan, le Turkménistan et l'Ouzbékistan, le Tadjikistan fait partie du groupe de pays représentés par la Suisse aux institutions de Bretton Woods, à la Banque mondiale et au Fonds monétaire international (FMI).

Les projets de coopération technique et financière visent à soutenir efficacement les réformes politiques, économiques et sociales au Tadjikistan et à atténuer les conséquences sociales du processus de réforme. Les projets de coopération technique ont pour objectif principal le transfert de connaissances professionnelles. Les projets visant à encourager un Etat de droit démocratique, les projets pour la santé publique et l'enseignement, la privatisation de l'économie des régions de montagne et des échanges culturels feront l'objet d'une stimulation particulière.

La coopération financière consistera principalement à financer des produits, appareils et matériels suisses destinés aux programmes prioritaires ainsi que des prestations liées et des transferts de connaissances spécialisées correspondantes. La coopération financière de la Suisse comprend également des aides à la balance des paiements et un allègement de la dette. La Suisse garantit un soutien financier principalement pour les projets importants d'infrastructures impossibles à financer de manière commerciale. Les projets dans le secteur de l'environnement se verront accorder une attention particulière ${ }^{24}$.

\section{Macédoine}

Le conseiller fédéral Joseph Deiss et le ministre macédonien des Affaires étrangères, Aleksandar Dimitrov, ont signé à Berne, le 26 octobre 1999, un accord de coopération technique et financière ainsi que d'aide humanitaire, qui règle les modalités du soutien suisse au processus de réforme engagé en Macédoine; il doit rester en vigueur pendant une période initiale de cinq ans.

Par cet accord, la Suisse vise à soutenir le processus de réforme engagé dans les pays de l'Est. Les projets de coopération technique visent avant tout à transmettre des connaissances techniques. Il est prévu d'encourager en particulier les projets de promotion des relations interethniques ainsi que les projets de réforme du secteur social, de privatisation de l'économie et de protection de l'environnement. Les échanges économiques et culturels, le commerce et les investissements seront également favorisés. Les dispositions de cet accord s'appliquent aussi à l'aide humanitaire apportée par la Suisse à la Macédoine.

La Suisse dispense son aide financière surtout en faveur de projets d'infrastructures importants qui ne pourraient être financés commercialement. Une attention 
particulière est portée aux projets mis en œuvre dans le secteur social, dans le domaine de l'énergie et dans celui de l'environnement ${ }^{25}$.

[D ASTM 1999, tableau récapitulatif des accords économiques entre la Suisse et les pays de l'Est, p. 213.

\subsection{DIPLOMATIE COMMERCIALE}

Un des volets de la politique économique extérieure de la Confédération concerne la diplomatie commerciale. L'encadré ci-dessous présente les principaux voyages de délégations économiques mixtes suisses (comprenant des représentants de l'administration fédérale et de l'économie privée) à l'étranger, mais également des voyages en Suisse de délégations d'Asie, d'Amérique latine ou de pays de l'Est. Dans la plupart des cas, les délégations sont menées par le conseiller fédéral en charge de l'Economie.

\section{$\square$ Diplomatie commerciale en 1999}

Il apparaît qu'en 1999, la diplomatie commerciale entre la Suisse et les pays d'Europe centrale et orientale a été intense. En ce qui concerne les pays asiatiques, les relations de la Suisse avec la Chine ont été marquées par la venue en Suisse du président chinois (mars 1999) et le voyage du conseiller fédéral Pascal Couchepin en novembre 1999 (voir ci-dessous «Relations économiques SuisseChine»). Des délégations économiques ont également fait le voyage en République populaire démocratique de Corée (Corée du Nord) en décembre 1998 ainsi qu'en Corée du Sud, en février 1999. Pascal Couchepin s'est aussi rendu en Arabie Saoudite, en février 1999. Quant à l'Amérique latine, les relations économiques bilatérales ont été soignées avec le Brésil (voyage de Pascal Couchepin en juillet 1999, voir ci-dessous «Relations économiques Suisse-Brésil») ainsi qu'avec le Chili (première réunion d'un groupe de travail Suisse-Chili en septembre 1999). A noter que pour cette année 1999, aucun voyage ayant pour objectif de développer des relations économiques n'a été effectué en Afrique.

\section{$\square$ Relations économiques Suisse-Chine}

1999 aura été une année très importante pour les relations helvético-chinoises puisque le président chinois, Jiang Zemin, est venu en Suisse en mars et que le conseiller fédéral Pascal Couchepin s'est rendu en Chine en novembre.

Visite du président chinois Jiang Zemin en Suisse

Le marché chinois attise les convoitises des entreprises suisses. Présente dans le pays depuis plus de vingt ans, la Suisse figure parmi les cinq plus gros investisseurs occidentaux en Chine. Elle totalise 2 à 3 milliards d'investissements directs, et plus de 500 sociétés suisses possèdent une société de production ou à tout le moins de distribution ${ }^{26}$. Les machines, la chimie et l'horlogerie sont les secteurs les plus présents en Chine. Le solde de la balance commerciale entre les deux pays est largement favorable aux Chinois, qui ont exporté pour 1,68 milliard de francs vers la Suisse en 1998. Quant aux importations helvétiques, elles

25. DDC et Seco, «Accord de coopération entre la Suisse et la Macédoine», Communiqué de presse, 26.10.99. 26. «Visite de Jiang Zemin, les dossiers que va plaider la Suisse», L'Agefi, 24.3.99. 
se montent à 809 millions $^{27}$. Mais, comme le relève Jacques Mermod, de la Société suisse des constructeurs de machines, dans un article de L'Agefi: «Les rapports entre la Chine et l'Occident sont ambigus. La main-d'œuvre chinoise est certes bon marché, mais la Suisse tient à ne pas trop transférer son savoir-faire. Du côté chinois, l'intérêt réside dans une croissance élevée des exportations tout en acquérant la technologie occidentale. ${ }^{28}$

A l'invitation du Conseil fédéral, Jiang Zemin, président de la République populaire de Chine, est venu en Suisse en visite d'Etat les 25, 26 et 27 mars 1999. Si, du côté suisse comme du côté chinois, on évoque cinquante ans de «bonnes relations» entre les deux pays (la Suisse a été l'un des premiers pays à reconnaître la République de Chine il y a cinquante ans), il n'en demeure pas moins que la visite du Chef d'Etat chinois a eu lieu à un moment où quelques points de tension apparaissent entre les deux pays. Il s'agit notamment du déficit commercial avec la Chine ${ }^{29}$, de la controverse autour de l'attribution de la GRE pour le barrage des Trois-Gorges, et du non-respect des droits de l'homme, soulevé par des ONG helvétiques (manque de liberté d'opinion, situation politique au Tibet et conditions de travail).

$\square$ ASTM 1997, barrage des Trois-Gorges, pp. 125-126.

ASTM 1998, idem, pp. 281-283.

Le Conseil fédéral, sous la conduite de la présidente de la Confédération, Ruth Dreifuss, a reçu le président chinois. Cette rencontre a été marquée par une manifestation de soutien au peuple tibétain, qui n'a pas été du goût de Jiang Zemin; celui-ci, furieux, a lancé au Conseil fédéral in corpore: "Vous venez de perdre un ami!» Malgré ce grave incident diplomatique, le voyage d'Etat du président chinois s'est poursuivi. Les deux premiers jours ont été consacrés à la diplomatie (relations bilatérales, politique et culture), la deuxième partie du voyage, prise en charge par l'Union suisse du commerce et de l'industrie - Vorort, étant nettement dévolue à l'économie (visites d'entreprises suisses et signatures de deux joint-ventures helvético-chinoises).

Dans un communiqué de presse ${ }^{30}$, l'administration fédérale annonçait que lors d'un entretien restreint entre la présidente de la Confédération et Jiang Zemin, la situation des droits de l'homme en Chine serait abordée, mais rien n'a filtré dans la presse sur le contenu et la nature de cet entretien. Des discussions de portée plus générale ont eu lieu, auxquelles se sont joints les conseillers fédéraux Ogi, Cotti et Couchepin. Elles ont concerné des questions de sécurité, la situation intérieure des deux pays, les aspects économiques globaux et les relations bilatérales entre les deux pays. La représentation suisse a notamment évoqué l'accès sans discrimination au marché chinois, la mise en œuvre de la protection de la propriété intellectuelle et l'ouverture dans les services (assurance-vie, banques et trafic aérien). A noter également que lors de cette visite, deux déclarations d'intention en matière d'éducation et des échanges culturels ont été signées ${ }^{31}$.

27. Direction générale des douanes, Statistiques annuelles du commerce extérieur, 1998.

28. "L'Empire du Milieu séduit toujours plus les entreprises helvétiques», L'Agefi, 25.3.99.

29. La Suisse a constaté une baisse de ses exportations vers la Chine en 1997-1998 (de 938 à 809 millions de francs), alors que les ventes dans le reste de l'Asie sont restées stables. Le déficit commercial avec la Chine a atteint un record en 1998 avec 873 millions de francs, sans tenir compte de Hong Kong (Le Temps, 25.3.99).

30. Départements fédéraux des affaires étrangères et de l'intérieur, Communiqué de presse, 25.3.99.

31. Le contenu de ces deux déclarations est précisé dans le Communiqué de presse mentionné dans la note n 30 . 


\section{Diplomatie commerciale - 1999}

\begin{tabular}{lll}
\hline Pays / Date & Nature de la visite & Objectifs et/ou résultats \\
\hline Asie & & \\
\hline $\begin{array}{l}\text { République populaire } \\
\text { démocratique de Corée } \\
\text { (Corée du Nord) }\end{array}$ & $\begin{array}{l}\text { Délégation économique mixte suisse, } \\
\text { sous la direction de l'ambassadeur } \\
\text { Nicolas Imboden (Seco). }\end{array}$ & $\begin{array}{l}\text { Objectifs: réunir des informations } \\
\text { "de première main" sur la situa- } \\
\text { tion et les perspectives prévalant } \\
\text { 12-15 décembre 1998 }\end{array}$ \\
& $\begin{array}{l}\text { en Corée du Nord; déterminer les } \\
\text { possibilités de développer les re- } \\
\text { lations économiques; discuter } \\
\text { de questions bilatérales en sus- } \\
\text { pens (arrières de paiement en par- } \\
\end{array}$ & $\begin{array}{l}\text { ticulier) ainsi que des bons offices } \\
\text { afin de réduire les tensions ré- }\end{array}$ \\
& gnant entre la Corée du Sud et la \\
& Corée du Nord.
\end{tabular}

Signature d'un accord de protection et promotion réciproques des investissements.

Corée du Sud Mission économique mixte sous la conduite du conseiller fédéral 7-11 février 1999 Pascal Couchepin. Une douzaine Renforcement des relations économiques et commerciales de représentants de l'économie privée étaient présents dans la délégation bilatérales tant sur le plan gouvernemental que sur celui de (Vorort, Sulzer, Novartis, ABB, UBS, Winterthur, Danzas, Fédération horlogère). l'économie privée.

Un Mémorandum d'entente sur le commerce et la coopération économique a été paraphé.

\begin{tabular}{lll}
\hline Arabie Saoudite & $\begin{array}{l}\text { Visite du conseiller fédéral } \\
\text { 19-23 février 1999 }\end{array}$ & $\begin{array}{l}\text { Renforcement des rapports } \\
\text { délégation officielle. }\end{array}$ \\
\hline
\end{tabular}

République populaire de Chine

\begin{tabular}{|c|c|c|}
\hline 25-27 mars 1999 & $\begin{array}{l}\text { A l'invitation du Conseil fédéral, } \\
\text { visite d'Etat de Jiang Zemin, } \\
\text { président de la République } \\
\text { populaire de Chine. }\end{array}$ & Voir ci-page précédente. \\
\hline 6-11 novembre 1999 & $\begin{array}{l}\text { Mission économique mixte } \\
\text { conduite par le conseiller } \\
\text { fédéral Pascal Couchepin. }\end{array}$ & \\
\hline \multicolumn{3}{|l|}{ Amérique latine } \\
\hline $\begin{array}{l}\text { Brésil } \\
\text { 5-8 juillet } 1999\end{array}$ & $\begin{array}{l}\text { Mission économique mixte } \\
\text { conduite par le conseiller fédéral } \\
\text { Pascal Couchepin. }\end{array}$ & $\begin{array}{l}\text { Voir ci-dessous «Relations } \\
\text { économiques Suisse-Brésil» } \\
\text { (pp. 290-291). }\end{array}$ \\
\hline $\begin{array}{l}\text { Chili } \\
\text { 23-24 septembre } 1999\end{array}$ & $\begin{array}{l}\text { Première réunion du groupe de travail } \\
\text { Suisse-Chili, à Berne, sous la conduite } \\
\text { de l'ambassadeur Pierre-Louis Girard. }\end{array}$ & $\begin{array}{l}\text { Entretiens sur les relations écono- } \\
\text { miques bilatérales et sur l'établis- } \\
\text { sement de relations préférentiel- } \\
\text { les entre le Chili et les pays de } \\
\text { l'AELE, le cas échéant dans le ca- } \\
\text { dre d'un accord de libre-échange. }\end{array}$ \\
\hline
\end{tabular}


Diplomatie commerciale - 1999

\begin{tabular}{|c|c|c|}
\hline Pays / Date & Nature de la visite & Objectifs et/ou résultats \\
\hline \multicolumn{3}{|l|}{$\begin{array}{l}\text { Europe } \\
\text { centrale et orientale }\end{array}$} \\
\hline $\begin{array}{l}\text { République de Moldova } \\
\text { 13-14 janvier } 1999\end{array}$ & $\begin{array}{l}\text { Visite officielle du président de la } \\
\text { République de Moldova à Berne. }\end{array}$ & $\begin{array}{l}\text { Signature d'un accord } \\
\text { de double imposition. }\end{array}$ \\
\hline $\begin{array}{l}\text { Croatie } \\
11-12 \text { mars } 1999\end{array}$ & $\begin{array}{l}\text { Visite officielle de travail du conseiller } \\
\text { fédéral Pascal Couchepin. }\end{array}$ & $\begin{array}{l}\text { Signature de deux accords: un } \\
\text { un accord de commerce et de } \\
\text { coopération économique et une } \\
\text { convention de double imposition. }\end{array}$ \\
\hline $\begin{array}{l}\text { Lettonie } \\
16 \text { mars } 1999\end{array}$ & $\begin{array}{l}\text { Visite du ministre de l'Economie } \\
\text { letton, Ainars Slesers. }\end{array}$ & $\begin{array}{l}\text { Entretiens sur les relations com- } \\
\text { merciales bilatérales, la coopéra- } \\
\text { tion multilatérale et le processus } \\
\text { d'intégration européenne. }\end{array}$ \\
\hline $\begin{array}{l}\text { Pologne } \\
22 \text { mars } 1999\end{array}$ & $\begin{array}{l}\text { Visite du conseiller fédéral } \\
\text { Kaspar Villiger à Varsovie, } \\
\text { accompagné d'une petite délégation } \\
\text { des représentants des milieux } \\
\text { bancaires et des assurances. }\end{array}$ & $\begin{array}{l}\text { Au centre des entretiens figurent } \\
\text { le remaniement de l'architecture } \\
\text { financière internationale, la colla- } \\
\text { boration au sein du FMl ainsi que } \\
\text { les relations bilatérales. }\end{array}$ \\
\hline $\begin{array}{l}\text { Bulgarie } \\
30 \text { avril } 1999\end{array}$ & $\begin{array}{l}\text { Visite d'une délégation économique } \\
\text { de Bulgarie conduite par le ministre } \\
\text { bulgare du Commerce et du Tourisme, } \\
\text { Valentin Vassiliev. }\end{array}$ & $\begin{array}{l}\text { Echange de vues et promotion des } \\
\text { relations économiques bilatérales } \\
\text { et des investissements. Discus- } \\
\text { sions avec des milieux écono- } \\
\text { miques et financiers suisses. }\end{array}$ \\
\hline $\begin{array}{l}\text { Kazakhstan } \\
\text { 9-10 septembre } 1999\end{array}$ & $\begin{array}{l}\text { Première réunion de la Commission } \\
\text { mixte Suisse-Kazakhstan, à Astana. } \\
\text { La délégation suisse, qui comprend } \\
\text { des représentants de l'économie } \\
\text { suisse est conduite par un membre } \\
\text { du comité de direction du Seco. }\end{array}$ & $\begin{array}{l}\text { Echange d'informations sur la } \\
\text { situation économique des deux } \\
\text { pays, les relations économiques } \\
\text { extérieures et l'évolution des } \\
\text { relations économiques bilatérales. }\end{array}$ \\
\hline $\begin{array}{l}\text { Russie } \\
28 \text { octobre } 1999\end{array}$ & $\begin{array}{l}\text { Cinquième réunion de la Commission } \\
\text { économique mixte Suisse-Russie, } \\
\text { à Berne. }\end{array}$ & $\begin{array}{l}\text { Organe consultatif des deux gou- } \\
\text { vernements créé par l'Accord de } \\
\text { commerce et de coopération signé } \\
\text { en } 1994 \text {. }\end{array}$ \\
\hline
\end{tabular}

Sources: DFE, Communiqués de presse parus en 1999.

Dans le contexte de sa coopération économique au développement avec la Chine, le Seco (à l'époque l'Office fédéral des affaires économiques extérieures - OFAEE) avait lancé en janvier 1998 le «Sino-Swiss Partnership Fund» (SSPF), destiné à mettre à la disposition de joint-ventures de PME suisse en Chine du capital-risque en monnaie locale ou en devises. La visite du président chinois a été l'occasion de signer les deux premières joint-ventures bénéficiant d'un financement au titre de ce fonds ${ }^{32}$. La délégation chinoise a également visité quelques grandes entreprises suisses (Novartis, Roche, ABB, Swatch).

32. Pour les détails concernant les partenaires suisses, se référer au Communiqué de presse du Seco (27.3.99). 


\section{Mission économique du conseiller fédéral Pascal Couchepin en Chine}

Le conseiller fédéral Pascal Couchepin s'est rendu en Chine à la tête d'une importante mission économique suisse, répondant à l'invitation chinoise lancée lors de la visite de Jiang Zemin au printemps. Initialement prévu en octobre, le voyage s'est finalement déroulé du 6 au 11 novembre $1999^{33}$. Selon les observateurs, l'incident qui avait marqué l'arrivée du président chinois sur la place Fédérale semble oublié (voir ci-dessus).

Pascal Couchepin a eu pour principaux interlocuteurs le premier ministre chinois, Zhu Rongji, qui tente de maintenir le cap de l'ouverture économique du pays, le ministre du Commerce extérieur et de la Coopération économique, Shi Guangsheng, et le ministre des Finances, Xian Huaicheng. Le conseiller fédéral était accompagné de hauts fonctionnaires du Seco. Quant au secteur privé, emmené par le président du Vorort, Andres Leuenberger, il comptait une vingtaine de représentants de l'économie suisse, dont Sulzer, ABB, SGS, Novartis, SAir-Group, Swatch et des groupes d'assurances.

La visite avait pour objectif de renforcer encore les liens économiques bilatéraux et de contribuer à développer de nouveaux domaines de coopération. Lors de ce voyage, Pascal Couchepin a ainsi pu inaugurer le bureau de représentation ouvert par Swiss Tourism, qui concrétise la première approbation chinoise donnée en la matière à un pays européen. Lors des différents entretiens, la Suisse a réaffirmé son soutien à l'entrée de la Chine au sein de l'Organisation mondiale du commerce, soulignant toutefois quelques problèmes à régler de la part des Chinois: droits de douane trop élevés, accès limité aux marchés des services et assurance du respect de la propriété intellectuelle. Lors de son entretien avec le premier ministre chinois, Pascal Couchepin a également évoqué la situation des droits de l'homme; sans se référer à des cas de prisonniers en particulier, il a néanmoins précisé que «la politique des droits de l'homme est essentielle et ne peut être séparée d'une bonne politique économique ${ }^{34}$. Aucun gros contrat n'a été signés lors de ce voyage, mais il s'agissait avant tout pour l'économie suisse d'intensifier les contacts bilatéraux et de préparer le terrain de futurs marchés, notamment celui des services.

\section{$\square$ Relations économiques Suisse-Brésil}

Le conseiller fédéral Pascal Couchepin s'est rendu du 5 au 8 juillet 1999 au Brésil à la tête d'une mission économique mixte.

Le Brésil est, de loin, le partenaire économique le plus important de la Suisse en Amérique latine en termes de commerce et d'investissements. Les grandes entreprises helvétiques de l'industrie alimentaire, pharmaceutique, chimique et mécanique y sont présentes depuis plusieurs décennies, y possèdent leur propre unité de production et y emploient environ 68'000 personnes. Pour la Suisse, le Brésil est la destination la plus importante, en Amérique latine, pour les investissements directs suisses. D'un point de vue brésilien, la Suisse est également perçue comme un partenaire important puisqu'elle se place au cinquième rang des investisseurs après les Etats-Unis, l'Allemagne, la Grande-Bretagne et le Japon.

33. DFE, «Mission économique du conseiller fédéral P. Couchepin en Chine», Communiqué de presse, 4.11.99.

34. «Pascal Couchepin panache d'un peu de droits de l'homme son menu pékinois», Le Temps, 10.11.99. 
L'objectif du voyage de la délégation mixte est «de resserrer les liens économiques et commerciaux bilatéraux aux plans gouvernemental et de l'économie privée». Parmi les buts spécifiques de la mission figurent la relance des pourparlers pour une convention de double imposition et la signature de deux déclarations d'intention, portant l'une sur le lancement d'un centre de promotion des technologies respectueuses de l'environnement, et l'autre sur l'élaboration du «Clean Development Mecanism» créé dans le cadre de la Convention sur les changements climatiques ${ }^{35}$.

CD ASTM 2000, dans la partie «Statistiques», voir le chapitre 1 («Commerce») et le sous-chapitre 2.6 («Investissements directs suisses à l'étranger»).

\section{SOURCES}

DFE, Communiqués de presse parus en 1999.

$\mathrm{CH}+6$, «Les entreprises et l'Etat. Attentes réciproques», n 106, décembre 1998.

La Vie économique, "Le Brésil et la globalisation», 2/99.

L'Agefi, série d'articles sur la visite du président chinois Jiang Zemin en Suisse, 26-29.3.99.

Le Temps, série d'articles sur la visite du conseiller fédéral Pascal Couchepin au Brésil, 5-8.7.99.

\subsection{RAPPORT SUR LES RELATIONS ENTRE LA SUISSE ET L'AFRIOUE DU SUD (1999)}

Le Conseil fédéral a publié, en octobre 1999, le rapport d'un groupe de travail interdépartemental sur les relations entre la Suisse et l'Afrique du Sud couvrant la période du début des années 50 à fin du régime de l'apartheid $(1994)^{36}$. Ce document examine le contexte juridique, économique et politique de ces relations; il ne traite pas des relations du service des renseignements suisse ${ }^{37}$, ni des activités des entreprises suisses en Afrique du Sud. Cette première étude ne se base que sur les sources de l'administration fédérale.

Les différents instruments de la politique suisse à l'égard de l'Afrique du Sud depuis le début des années 50, tels que la mise en œuvre d'un plafond pour 1'exportation de capitaux, la surveillance statistique des flux commerciaux de certains produits ainsi que l'application de l'embargo sur les armes, sont évalués, de même que l'attitude de la Suisse concernant les investissements, la coopération au développement et l'endettement.

Pour la première fois, un rapport officiel, approuvé par le Conseil fédéral, laisse entendre que l'attitude de la Suisse à partir de 1985 n'était plus défendable, du point de vue tant moral que politique: «On doit constater qu'une attitude aussi prudente à l'égard de mesures coercitives que celle suivie dans les années 80 face à l'Afrique du Sud ne pourrait, actuellement, être expliquée par des motifs de politique de neutralité» (p. 22). Toutefois, les experts concluent que la Suisse n'est pas devenue une plaque tournante pour des opérations visant à esquiver les sanctions internationales contre le régime de l'apartheid. Le rapport rappelle

35. DFE, «Mission économique du conseiller fédéral Pascal Couchepin au Brésil du 5 au 8 juillet 1999», Communiqué de presse, 28.6.99.

36. DFE, «Les relations entre la Suisse et l'Afrique du Sud», Communiqué de presse, 1.10.99. Le rapport est disponible sur le site Internet www.dfe.admin.ch/dynamic/Presse_Rohstoff/CH_RSA/F/af_sud_1f.htm.

37. Cette question a fait l'objet d'un rapport de la Délégation des commissions de gestion intitulé Le rôle des services de renseignements suisses dans le cadre des relations entre la Suisse et l'Afrique du Sud. Le rapport conclut que les soupçons relatifs à une participation supposée d'autorités militaires suisses à la mise en place d'un programme d'armes biologiques et chimiques du régime d'apartheid sud-africain ne se sont pas confirmés. 
également que les parlementaires ont été amenés à débattre à de nombreuses reprises sur la politique gouvernementale à l'égard de l'Afrique du Sud, puisqu'une estimation provisoire fait état de 160 interventions parlementaires pendant la période analysée. Certaines d'entre elles réclamaient des sanctions économiques, mais aucune n'a recueilli un soutien suffisant. Et d'en conclure que «dans ces circonstances, il n'est nullement exagéré de dire que, dans ses grandes orientations, la politique du Conseil fédéral à l'égard de l'Afrique du Sud a reçu un soutien parlementaire massif» (p. 22).

Un des mérites du rapport réside dans l'encouragement à conduire des études complémentaires, afin de pouvoir porter un jugement global sur la politique de la Suisse à l'égard de l'Afrique du Sud. Il s'agirait d'approfondir certaines questions concernant le comportement de la Suisse, le rôle des entreprises privées suisses, mais également le comportement des autres pays occidentaux, plus spécifiquement en relation avec les sanctions économiques. Dans ses conclusions, le groupe de travail propose que ces questions fassent l'objet d'un programme de recherche du Fonds national suisse. Le Conseil fédéral a donné mandat aux offices concernés de préparer l'intégration de telles recherches au PNR42, «Fondements et possibilités de la politique extérieure suisse». 